\title{
Motional Enhancement of Exciton Magnetic Moments
}

\author{
A. Litvinov, V. Kochereshko \\ A.F. Ioffe Physico-Technical Institute, RAS \\ 194021 St. Petersburg, Russia \\ R.T. Cox, L. Besombes, H. Mariette, H. Boukari \\ Laboratoire de Spectrométrie Physique \\ Université Joseph Fourier de Grenoble, France

\section{LOGINOV} \\ Institute of Physics, St. Petersburg State University, St. Petersburg, Russia

\section{J.J. Davies, L.C. Smith And D. Wolverson} \\ Department of Physics, University of Bath, Bath BA2 7AY, UK \\ The phenomenon in which giant enhancement of exciton magnetic mo- \\ ments occurs due to translational motion was found for light hole excitons \\ in $\mathrm{ZnTe} / \mathrm{ZnMgTe}$ quantum well structures. Decreasing diamagnetic shifts as \\ the number of the exciton quantized state increases were found for the first \\ time. \\ PACS numbers: 71.35.Cc, 71.35.Ji, 71.36.+c, 78.20.Ls
}

\section{Introduction}

In exciton magneto-optics the exciton center of mass motion is usually neglected. This is caused, first of all, by the fact that, generally, only excitons with very small wave vector $K=\frac{\omega}{c} n \propto 10^{5} \mathrm{~cm}^{-1}$ and small kinetic energy participate in optical experiments. Spectroscopy of moving excitons is difficult due to the fact that such excitons cannot interact with light.

The magnetic moment of an exciton at rest is determined by sum of the hole and electron magnetic moments as well as, in some cases, by the orbital moments. The exciton center of mass motion can significantly change or even result in new magnetic properties of the exciton. The exciton center of mass motion can combine with the internal motion and this will result in specific magnetic properties of the 
excitons. The first experimental observation of the mutual effects of magnetic field and exciton motion was reported in classical papers [1,2]: in these papers exciton movement perpendicular to magnetic fields leads to emergence of quasi-electric field in the system of the exciton center of mass. The phenomenon of magnetic field induced spatial dispersion was observed in thin CdSe and GaAs crystals [3, 4]. Detailed analysis of mutual magnetic field and wave vector effects on the exciton reflectivity spectra in zinc-blende crystals has been published in [5]. Recently some of the present authors have observed a new effect of "motional enhancement of the exciton magnetic moment" (MEM). In this effect, the exciton magnetic moment (exciton $g$-factor) increases for the moving exciton [6] as the translational kinetic energy gets larger.

An excellent opportunity for studying exciton states with large kinetic energy is provided by quantum well (QW) structures. In such structures the quantized exciton states correspond to states with different kinetic energies. The higher the sequence number of the state, the higher is the kinetic energy of the exciton [7].

In the present paper we have studied reflectivity spectra from QW structures in magnetic fields in the Faraday $H\|K\|[100]$ geometry. In addition to the work in paper [6], we study the MEM effect not only on a heavy hole exciton but on a light hole, too. In this study we used quantum wells, but it appears that the observed phenomena are general and relate to bulk zinc-blende type crystals. The use of quantum wells in this research allowed us only to study the states with high kinetic energies.

\section{Experiment}

$\mathrm{ZnTe} / \mathrm{Zn}_{0.9} \mathrm{Mg}_{0.1} \mathrm{Te}$ and $\mathrm{ZnTe} / \mathrm{Zn}_{0.95} \mathrm{Mg}_{0.05} \mathrm{Te}$ heterostructures with wide quantum wells of 50 and $100 \mathrm{~nm}$ width, respectively, have been studied. The samples were grown by MBE on ZnTe [100] substrates, covered by $1100 \mathrm{~nm}$ $\mathrm{Zn}_{0.95} \mathrm{Mg}_{0.05}$ Te buffer layer. These samples were strained due to lattice mismatch between QW and barrier layer that leads to a splitting of the heavy and light hole bands in the QW. In our particular case, the light hole band was $15 \mathrm{meV}$ lower in energy than the heavy hole band. Reflectivity spectra were measured in magnetic fields $H$ up to $8 \mathrm{~T}$ in the Faraday geometry $H\|K\|[100]$ at liquid helium temperature $4.2 \mathrm{~K}$.

\section{Results and discussions}

Reflectivity spectra taken from the $100 \mathrm{~nm}$ QW structure are presented in Fig. 1. There are two different parts in the spectrum: the first part from 2.4 to $2.41 \mathrm{eV}$ corresponds to the exciton states in the barrier material $\mathrm{ZnMgTe}$, the second part from 2.37 to $2.4 \mathrm{eV}$ corresponds to the exciton states in the QW. The excitons as a whole are quantized in the wide QW, causing sharp maxima and minima in the reflectivity spectrum. We designate these spectral features by integer numbers $N$ (Fig. 1). There are two different types of features in this 

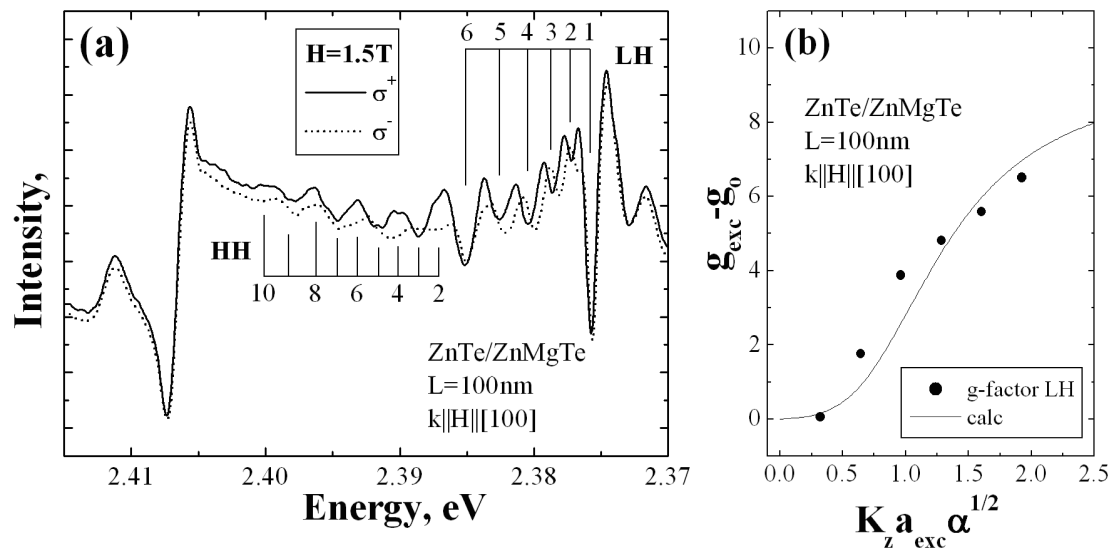

Fig. 1. (a) Reflectivity spectrum taken from the $\mathrm{ZnTe} / \mathrm{ZnMgTe} 100 \mathrm{~nm}$ quantum well in magnetic field of $1.5 \mathrm{~T}$ in two circular polarizations $\sigma^{+}$and $\sigma^{-}$. (b) Dependence of the exciton $g$-factor on the dimensionless exciton center of mass wave vector. Symbols are experimental, the curve is calculated with formula (1).

spectrum. In the range from 2.375 to 2.385 these features correspond to the quantized states of light excitons (LH formed by an electron and light hole). In the range from 2.85 to 2.40 , they correspond to the quantization of heavy excitons $\mathrm{HH}$.

From the spectra it is obvious that the magnitude of the Zeeman splitting for light exciton states increases as the quantization number grows. From the spectra we extracted different excitonic parameters, such as the $g$-factor and the diamagnetic constant $d[8]$. Figure 1b shows the dependence of the light exciton magnetic moment on its wave vector. This dependence is qualitatively similar to the previously published heavy exciton dependence [6]. It can be described by the same formula as in [6], namely

$$
g_{\mathrm{exc}}-g_{0}=\frac{1}{2} G\left(1-\frac{1}{\sqrt{1+\alpha^{2}\left(a_{\mathrm{B}} K\right)^{4}}}\right) .
$$

Here $a_{\mathrm{B}}$ is the exciton Bohr radius and $K$ is the exciton translational wave vector. For the present material, the constants $G=17, \alpha=2.64$ were determined from experiment. These data give us the value of the constant at cubic in wave vector term in the valence band dispersion of ZnTe $\gamma_{\nu} a_{2}=3.42 \mathrm{~nm}^{3} \mathrm{eV}$.

Figure 2a shows a set of dependences of the value of the diamagnetic shift $\left(\Delta E=d H^{2}\right)$ on the square of magnetic field for different exciton quantized levels. It is clear that the diamagnetic constant $d$ depends on the quantization number and that the dependence remains nearly quadratic in $H$ for all levels. Figure 2b shows the dependence of the diamagnetic constant $d$ on the quantization number of the exciton center of mass level or on the exciton wave vector $K(K=N \pi / L)$.

With the present accuracy we can consider this dependence as linear, so that $d=d_{0}-\beta K$. Here $d_{0}$ is the diamagnetic constant for the exciton at rest 

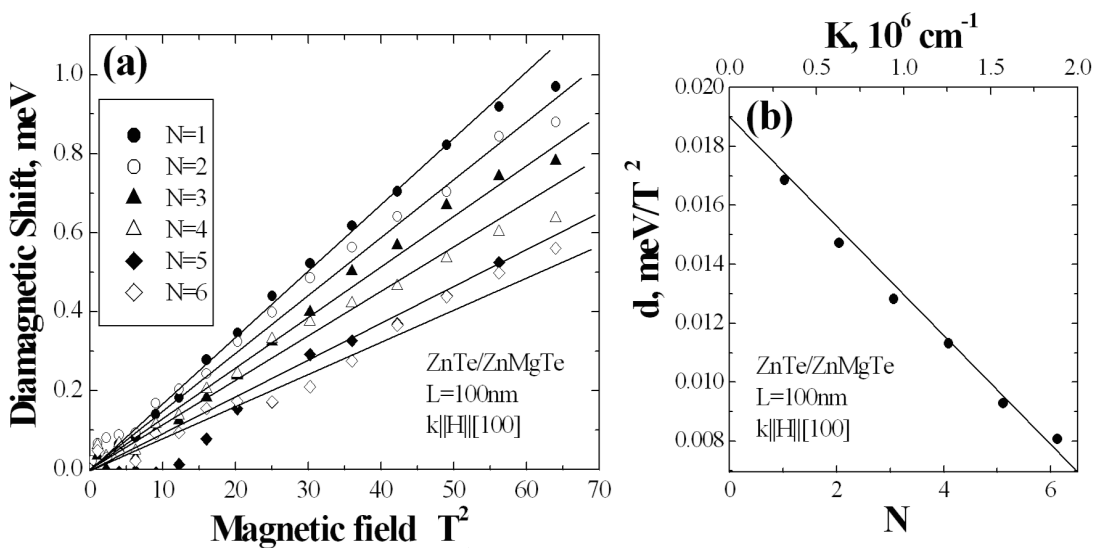

Fig. 2. (a) Dependence of exciton diamagnetic shift on the square of magnetic field for different numbers of quantized states. (b) Diamagnetic constant $d$ as a function of quantized state number.

and $\beta$ is some constant. From our data we determine $d_{0}=0.019 \mathrm{meV} / \mathrm{T}^{2}$ and $\beta=5.75 \times 10^{-3} \mathrm{~nm} \mathrm{meV} / \mathrm{T}^{2}$ for ZnTe.

We suppose that the reason for such a dependence of the diamagnetic shift on the wave vector is the presence of cubic in wave vector terms in the valence band Hamiltonian, the same terms that give contribution to the exciton $g$-factor [6]. Indeed, the only terms in the exciton Hamiltonian which give diagonal contribution $\propto H^{2} K$ into the exciton energy are cubic wave vector terms [9]:

$$
\delta H_{\nu 3}(k)=\gamma_{\nu} a_{2} \sum_{i} J_{i}^{3} \kappa_{i} .
$$

Here $J_{i}$ are the matrices for $J=3 / 2$, and $\kappa_{i}=k_{i}\left(k_{i+1}^{2}-k_{i+2}^{2}\right)$, where $k_{i}(i=$ $x, y, z)$ are the components of the $\boldsymbol{k}$ vector. This Hamiltonian can give nonzero contribution in the second order of perturbation for exciton quantized states. We can obtain the value $\gamma_{\nu} a_{2}$ from the diamagnetic shift data. It is found to be $\gamma_{\nu} a_{2} \approx 3.2 \mathrm{~nm}^{3} \mathrm{eV}$ that is close to ones obtained from $g$-factor at least in the order of magnitude.

Here we should mention that $\mathrm{CdTe}$ and ZnSe are similar in many respects. Consequently it is natural that the values of $\gamma_{\nu} a_{2}$ are close in these materials (in CdTe $\left.\gamma_{\nu} a_{2}=2.01 \mathrm{~nm}^{3} \mathrm{eV}[6]\right)$. At the same time the value $\alpha$ in our case of the light hole exciton is 4 times bigger than the one of the heavy hole exciton in CdTe. This difference comes from the different matrix elements in $J^{3}$ matrix for light and heavy holes.

\section{Conclusions}

Reflectivity spectra taken from $\mathrm{ZnTe} / \mathrm{ZnMgTe}$ wide QWs were studied in magnetic fields in the Faraday geometry. The phenomenon of the "giant increase in exciton magnetic moments" has been observed and in this case studied for the 
light hole exciton. A dependence of exciton diamagnetic shift on the exciton center of mass wave vector has been found. This gives us independent measurements of cubic in wave vector terms in the valence band in zinc-blende semiconductors.

\section{Acknowledgments}

This work was supported in part by grants of the RFBR, the Presidium RAS, the Royal Society, EPSRC (UK) and CNRS.

\section{References}

[1] D.G. Thomas, J.J. Hopfield, Phys. Rev. 124, 657 (1961).

[2] E.F. Gross, B.P. Zakharchenia, O.V. Konstantinov, Sov. Phys.-Solid State 3, 305 (1961).

[3] E.L. Ivchenko, V.P. Kochereshko, G.V. Mikhailov, I.N. Uraltsev, JETP Lett. 37, 137 (1983).

[4] O.V. Gogolin, V.A. Tsvetkov, E.G. Tsitsishvilli, JETP 87, 1038 (1984).

[5] K. Cho, S. Suga, W. Deybrodt, F. Willmann, Phys. Rev. B 11, 1512 (1975).

[6] J.J. Davies, D. Wolverson, V.P. Kochereshko, A.V. Platonov, R.T. Cox, J. Cibert, H. Mariette, C. Bodin, C. Gourgon, E.V. Ubyivovk, Yu.P. Efimov, S.A. Eliseev, Phys. Rev. Lett. 97, 187403 (2006).

[7] V.A. Kiselev, B.S. Razbirin, I.N. Uraltsev, Phys. Status Solidi B 72, 161 (1975) and H. Tuffigo, R.T. Cox, N. Magnea, Y. Merle d'Aubigné, A. Million, Phys. Rev. B 37, 4310 (1988).

[8] D.K. Loginov, E.V. Ubyivovk, Yu.P. Efimov, V.V. Petrov, S.A. Eliseev, Yu.K. Dolgikh, I.V. Ignatiev, V.P. Kochereshko, A.V. Selkin, Phys. Solid State 48, 2100 (2006).

[9] E.L. Ivchenko, G.E. Pikus, Superlattices and Other Heterostructures, in Springer Series in Solid-State Sciences, Vol. 110, Springer, Berlin 1995, p. 73. 